\title{
Cambios en la Arquitectura Indígena de la Región de Madre de Dios, Perú (años 1990)
}

Tanith Olórtegui del Castillo de Rummenhoeller - Arquitecta, alumna del Curso de Pos Graduación Maestría en Desarrollo Regional PPG-MDR de la Universidade Federal do Acre, UFAC.

\section{Resumo}

La existencia de una diversidad étnica y cultural en el Perú, en especial en la Amazonía debe ser tomada en cuenta para entender las diferencias de habitabilidad y de vivienda. La presión de la sociedad nacional sobre las etnias amazónicas ha originado cambios fundamentales em su modo de vida, derivando en la pérdida de sus valores culturales. Los orígenes de estos cambios se hallan en el trasfondo histórico así como en los diferentes factores e agentes de cambio de la sociedad dominante. En numerosos casos son los indígenas quienes aceleran mudanzas por propia decisión, muchas veces sin pensar en las desventajas que estos lês ocasionan. Un reflejo muy importante de dicho proceso se aprecia en la progresiva transformación de sus viviendas, en el uso de materiales y formas ajenas a su cultura así como modificaciones en su distribución espacial que traen también como consecuencia alteraciones en su confort interno y condiciones de salubridad. Presentamos aquí un enfoque diferente desde los campos de la arquitectura y la antropología, ampliando el conocimiento en el campo de la vivienda indígena en la Amazonía peruana en particular y en la Pan Amazonia em general, introduciendo en el debate sobre el tema la dimensión cultural de la vivienda y su diversidad en la búsqueda de nuevas alternativas de cambio, a modo de obtener uma comprensión integral de la problemática habitacional indígena.

\section{Palavras-chave}

Vivienda Indígena; Cambio Cultural; Comunidades Indígenas; Madre de Dios.

\begin{abstract}
The existence of ethnic and cultural diversity in Peru, especially in the Amazon should be taken into account to understand differences and habitable housing. The national society pressure on Amazonian ethnic groups has led to fundamental changes in their way of life, resulting in the loss of their cultural values. The origins of these changes are in the historical background as well as in different factors and agents of change in the dominant society. In many cases the natives themselves accelerate the movements by choice, often without thinking about the disadvantages that they cause on them. An important reflection of that process is seen in the gradual transformation of their homes, the use of materials and forms alien to their culture and changes of the spatial distribution, which result in changes of its internal comfort and sanitation. We present here a different approach from the fields of architecture and anthropology, to expand the knowledge in the field of indigenous housing in the Peruvian Amazon in particular and in general in the Pan-Amazon, introducing the debate of the cultural dimension of housing and diversity in the search for new alternatives for change and for an integral understanding of the issue of indigenous housing.
\end{abstract}

\section{Keywords}

Indigenous Housing, Cultural Change, Indigenous Communities, Madre de Dios. 


\section{INTRODUCCIÓN}

La presión de la sociedad nacional sobre las etnias amazónicas ha originado câmbios fundamentales en su modo de vida, derivando en la pérdida de sus valores culturales. Los orígenes de estos cambios se hallan en el trasfondo histórico así como en los diferentes factores e agentes de cambio de la sociedad dominante. En numerosos casos son los indígenas quienes aceleran mudanzas por propia decisión, muchas veces sin pensar en las desventajas que estos lês ocasionan. Un reflejo muy importante de dicho proceso se aprecia en la progresiva transformación de sus viviendas, en el uso de materiales y formas ajenas a su cultura así como modificaciones en su distribución espacial que traen también como consecuencia alteraciones en su confort interno y condiciones de salubridad.

En el Perú no encontramos antecedentes sobre estudios de viviendas contemporáneas em comunidades indígenas; los antropólogos se han concentrado en la búsqueda de rasgos tradicionales en la vivienda como parte de la cultura tradicional de estas etnias que más son contribuciones históricas al estudio de las mismas y para los arquitectos éstos no son considerados propiamente como expresiones arquitectónicas provistas de mayor contenido para ser estudiadas. Dentro de este marco general, presentamos aquí un enfoque diferente desde ambos campos, pretendemos contribuir a ampliar el conocimiento en el campo de la vivienda indígena en la Amazonía peruana en particular y en la Pan Amazonia en general, introduciendo en el debate sobre el tema la dimensión cultural de la vivienda y su diversidad en la búsqueda de nuevas alternativas de cambio, a modo de obtener una comprensión integral de la problemática habitacional indígena.

La investigación de campo del presente estudio se llevó a cabo entre 1988 y 1990 com apoyo financiero del Consejo Nacional de Ciencia y Tecnología del Perú (CONCYTEC) bajo el auspicio del Instituto Indigenista Peruano (IIP).

\section{ENTORNO GEOGRÁFICO}

La subregión de Madre de Dios se encuentra situada en la zona Sur Oriental del Perú, comprendiendo diversas zonas ecológicas de la sierra, ceja de selva y selva baja. Tiene uma extensión de 78,402.71 $\mathrm{Km}^{2}$. Limita por el norte con la Región de Ucayali y la República Federativa del Brasil (Estado de Acre), por el este con el mismo Estado de Acre y la República 
de Bolivia (Departamento de Pando), por el sur con la subregión de Puno y por el suroeste com la subregión de Cusco.

La hoya hidrográfica del Madre de Dios constituye una sub-cuenca del río Madeira que desemboca en el Amazonas por su margen derecha. De acuerdo a la clasificación presentada por Ríos et al. (1986) la subregión Madre de Dios cuenta con 12 zonas de vida y 4 zonas de vida em transición que poseen una alta diversidad de flora y fauna. Las zonas de vida predominantes em el ámbito de este estudio y sus características son las siguientes:

Tabla 1: Zonas de vida de la Región de Madre de Dios.

\begin{tabular}{|c|c|c|c|}
\hline Zona de vida & $\begin{array}{c}\text { Altitud } \\
\text { (m.s.n.m.) }\end{array}$ & \begin{tabular}{|c|} 
Precipitación \\
(mm/año)
\end{tabular} & Comunidades \\
\hline $\begin{array}{l}\text { Boque húmedo- } \\
\text { Subtropical (bh-S) }\end{array}$ & $220-300$ & $1200-1960$ & \multirow{2}{*}{$\begin{array}{l}\text { Palma Real, Infierno, } \\
\text { Puerto Arturo, Boca } \\
\text { Pariamanu, Boca } \\
\text { Inambari. }\end{array}$} \\
\hline $\begin{array}{l}\text { Bosque húmedo- } \\
\text { Tropical (bh-T) } \\
\end{array}$ & $200-350$ & $1900-3000$ & \\
\hline $\begin{array}{l}\text { Bosque muy húmedo- } \\
\text { Subtropical (bmh-S) }\end{array}$ & $200-400$ & $4000-4500$ & $\begin{array}{l}\text { San José del Karene, } \\
\text { Diamante }\end{array}$ \\
\hline $\begin{array}{l}\text { Bosque muy húmedo- } \\
\text { Premontano Tropical } \\
\text { (bmh-PT) }\end{array}$ & $400-700$ & $3000-3500$ & Shintuya, Palotoa \\
\hline
\end{tabular}

No existen mediciones de temperatura representativas según las diferentes zonas de vida pero según datos de ONERN (1972) la temperatura media anual de la región es de $26^{\circ} \mathrm{C}$ y la humedad relativa media anual de $75 \%$. Las temperaturas máximas alcanzan los $39^{\circ} \mathrm{C}$, pudiendo descender la mínima hasta $6^{\circ} \mathrm{C}$ en los meses de mayo a setiembre durante los días del "surazo". Este fenómeno meteorológico se debe al paso de ondas de aire frío provenientes del anticiclón del Atlántico Sur.

Con respecto a la fisiografía en el ámbito de estudio se puede determinar (ONERN, 1972) dos principales unidades fisiográficas: paisaje aluvial y paisaje colinoso. El paisaje aluvial está conformado por sedimentos tanto recientes (llanuras de inundación) como antiguos (llanura de sedimentación) que se caracterizan principalmente por su topografía relativamente plana $(0-5 \%)$.

\section{UNIVERSO POBLACIONAL DE MADRE DE DIOS}

Madre de Dios está habitada por aproximadamente 17 etnias que pertenecen a 5 familias lingüísticas. Las etnias más importantes son; 
Amarakaeri, Huachipaeri (familia lingüística Harakmbut), Esse'Eja (Tacana), Piro, Machiguenga (Arawak), Amahuaca, Yaminahua, Nahua, Shipibo-Conibo (Pano) y Kichwa Runa (Quechua del Oriente). La población indígena en Madre de Dios llegaba en los años 1990 a aproximadamente 5,000 habitantes (que hoy suman alrededor de 6,000 personas) conformando unas 35 comunidades nativas, 20 de cuales tienen posesión legal sobre cierta cantidad de territorios colectivos. Para este estudio se ha elegido lãs siguientes 9 comunidades representativas: Palma Real, Infierno (Esse'Eja), Boca del Inambari, San José del Karene (Amarakaeri), Shintuya (Amarakaeri, Huachipaeri), Puerto Arturo (Kichwa Runa), Palotoa-Teparo (Machiguenga), Diamante (Piro) y Boca Pariamanu (Amahuaca).

La mayoría de las comunidades nativas vive desde hace años en contacto con La sociedad nacional, combinando las actividades de subsistencia como la agricultura de roza y quema, caza, pesca, recolección, con actividades orientadas al mercado como la extracción de madera, lavado de oro, recolección de nueces de Pará y la ganadería en pequeña escala.

La población total de la sub-región aumentó de 14,000 a más de 50,000 habitantes en los últimos 20 años, debido a la afluencia de migrantes procedentes de la sierra; de los cuales

muchos constituyen una población flotante en los lavaderos de oro. Los migrantes en muchos casos invaden tierras de las comunidades nativas causando graves problemas sociales. Para defender con mayor eficacia sus derechos territoriales, de educación y salud, la mayoría de lãs comunidades se ha organizado en la Federación Nativa del río Madre de Dios y Afluentes (FENAMAD).

\section{CONCEPTO DE VIVIENDA INDÍGENA}

La existencia de una diversidad étnica y cultural en el Perú, en especial en la Amazônia debe ser tomada en cuenta para entender las diferencias de habitabilidad y de vivienda. Norberg Schulz (1971) señala que el espacio existencial representa el modo como el hombre se sitúa em el mundo, el cómo reside; por otro lado la creación de un espacio arquitectónico significa La integración de una forma intencionada de vida en el ambiente, idealmente debe existir uma relación entre el espacio existencial y el espacio arquitectónico, el uno debe ser la consecuencia lógica del otro. En este contexto, el espacio arquitectónico nativo es reflejo directo del modo de vida indígena. Los nativos al construir sus viviendas traducen sus esquemas 
personales em estructuras arquitectónicas concretas; de ahí la importancia de evaluar los cambios que ellos mismos realizan en sus viviendas, los cuales son muchas veces copias de estructuras que no responden a su espacio existencial, esto conlleva a modificaciones posteriores siempre en uma constante búsqueda de mejores respuestas arquitectónicas que más respondan a su forma particular de residencia.

Es vital remarcar que el concepto de vivienda de la población nativa difiere del nuestro. La vivienda nativa concebida como "unidad de habitación" es un complejo de edificaciones y espacios utilizados por el grupo familiar para su actividad constante. Cumple con una cantidad de roles, es el alojamiento familiar, dotado de los espacios de habitación y de alimentación. También es el almacén de diversos productos y alimentos. La vivienda es el lugar de trabajos artesanales, así como procesamientos de los productos de la chacra (el secado de tabaco, guisador, etc.).

La vivienda nativa presenta pues gran flexibilidad en el uso de sus ambientes, debido a las múltiples actividades que en ella se realizan no es posible encasillar cada actividad con um ambiente determinado. Es preciso entender esto y evitar la visión meramente funcionalista al interpretar términos como "casa", "cocina”, "dormitorio", "estar", "depósito", que son empleados en este estudio como simple denominación (por la actividad predominante, mas no excluyente, que se realiza en los espacios).

La disponibilidad de recursos para la construcción de la vivienda es un factor definitivo em su configuración física. Entre los nativos la distinción entre la utilización de uno u otro material influye en el status de la vivienda. Esto último ligado a la existencia de una distinta noción de uso del espacio es un aspecto de particular importancia para este trabajo. Mencionamos a continuación algunas características muy específicas que reflejan dicho aspecto:

- Espacialmente la vivienda nativa es ilimitada.

- Las actividades de sus moradores no se circunscriben al predio.

- El mayor uso y vivencia de la vivienda se da fuera de la edificación, especialmente en lãs áreas sociales.

- Existe gran flexibilidad en las áreas de edificación, es decir que éstas pueden cambiarse o adaptarse según la necesidad presente.

- Su uso principal es como espacio para dormir y cocinar, así como espacio destinado a depósito del hogar. 
- No significa una inversión inmobiliaria, según las reglas sociales indígenas no existe el derecho de propiedad individual sobre la tierra sino el de su usufructo.

- La vivienda es concebida como un bien temporal, práctico, cuya vida útil es de mediano plazo (5 a 8 años).
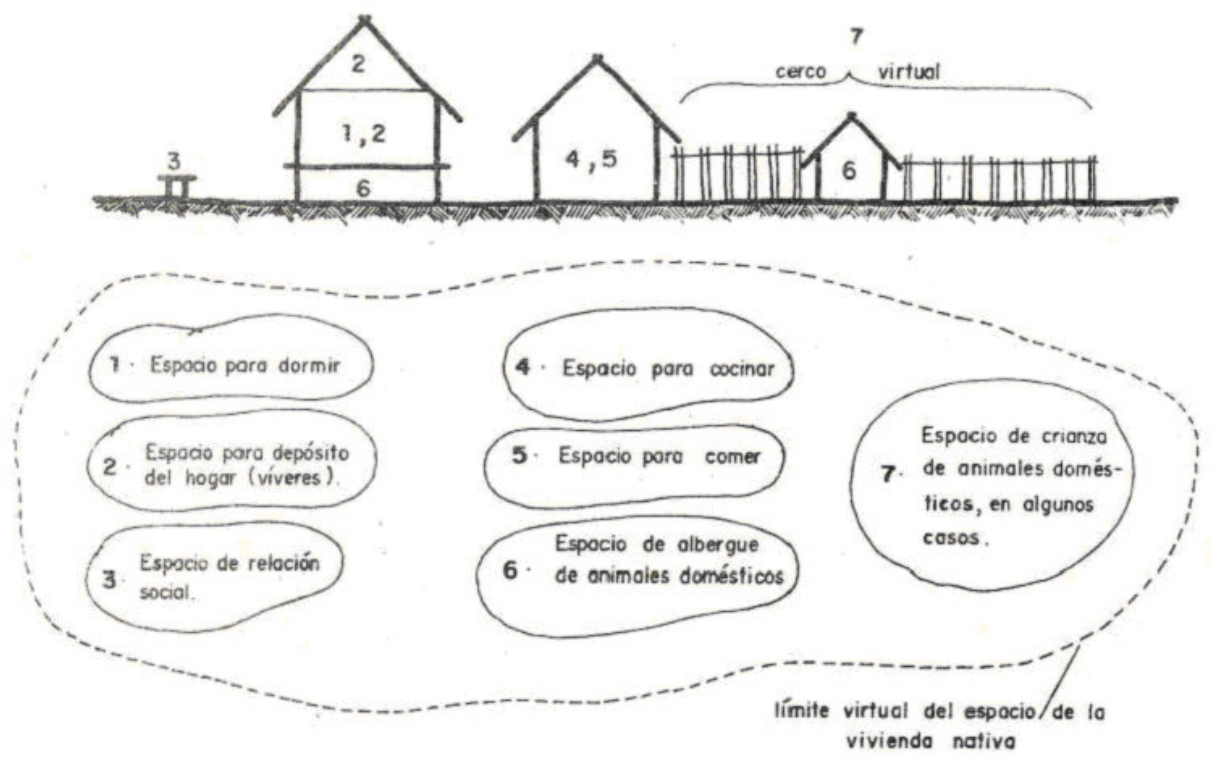

Gráfico 1: Composición espacial de la vivienda indígena.

\section{TIPOLOGÍA DE LA VIVIENDA INDÍGENA}

Las diferentes concepciones de tipo han experimentado una gradual evolución em el tiempo hacia una mayor precisión y profundidad. Sin embargo este proceso no ha conducido necesariamente a una opinión consensual que actualmente permita y facilite la aceptación, entendimiento y comunicación de lo que de él se entiende. Esta diversidad de interpretaciones nos compromete en este caso a establecer cuál es la versión más adecuada y que mejor sirva a los objetivos de este proyecto porque de él derivará el desarrollo de una parte importante Del mismo.

Coincidimos con la posición de Aldo Rossi (1979) quien "concibe el tipo como algo concreto y ya existente, unido a la forma y al modo de vida de los pueblos". Para él "es um enunciado lógico que se antepone a la forma y la constituye; es permanente y complejo y no uma simple invención sino algo sobre lo cual el arquitecto interviene sólo para progresar en la situación actual". De manera particular nos basamos en 
nociones organizativas del espacio habitable, el tipo al cual nos referimos es el del registro y clasificación de lo existente que combinado con los factores condicionantes locales nos permite delinear una imagen conceptual recomendable que corresponde a esta región.

Queremos expresar que en este trabajo las tipologías que se expondrán corresponden a enunciados lógicos que se anteponen a modelos, referidos a la forma y al modo de vida de los pueblos indígena, condicionados por el contexto físico y los recursos del lugar. Basándonos em estos conceptos hemos definido dos tipos: T-1 y T-2. El primero correspondiente a viviendas de un solo volumen (casa-cocina) y el segundo conformado por viviendas de dos volúmenes (casa y cocina).

Para este estudio se desarrolló un esquema propio enfatizando los estados tecnológicos de transformación de la vivienda indígena adecuándolo a nuestra zona de estudio, en base a um esquema de Fonseca y Saldarriaga (1980, p. 31), aplicado a viviendas rurales en Colombia. Definimos cuatro categorías básicas de vivienda según su estado tecnológico:
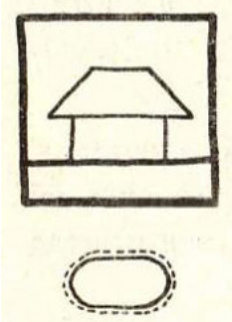

AUTOCTONO

A
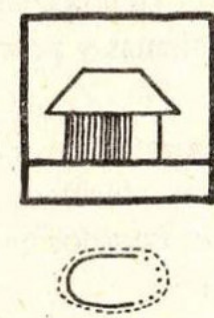

TRANSICION AUTOCTONAL

TAT
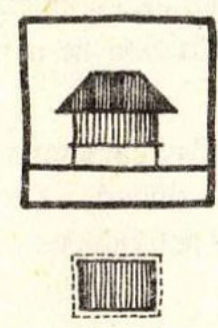

TRADKIONAL.

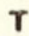
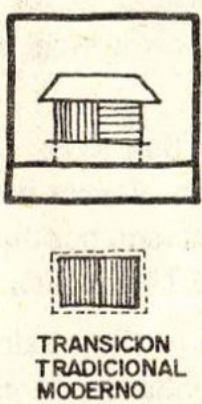

TrM
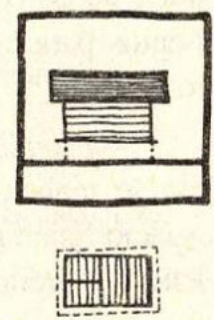

MODERNO

M

Gráfico 2: Estados tecnológicos de transformación de la vivienda indígena.

- Viviendas Autóctonas (A): Son las viviendas construidas con materiales obtenidos del lugar, que no requieren de tipo alguno de elaboración previa a su utilización. Son viviendas con estructura de madera rolliza, cerramientos de hojas de palmera en la cubierta ("shebón", "palmichi”, "shapaja"), caña brava, "huasaî" o "pona" en las paredes; uniones con soga vegetal "tamshi", "atadijo", etc., piso de tierra apisonada. Viviendas de planta circularovalada, multifamiliares, en algunos casos con divisiones interiores para cada familia, em otros casos sin divisiones. 


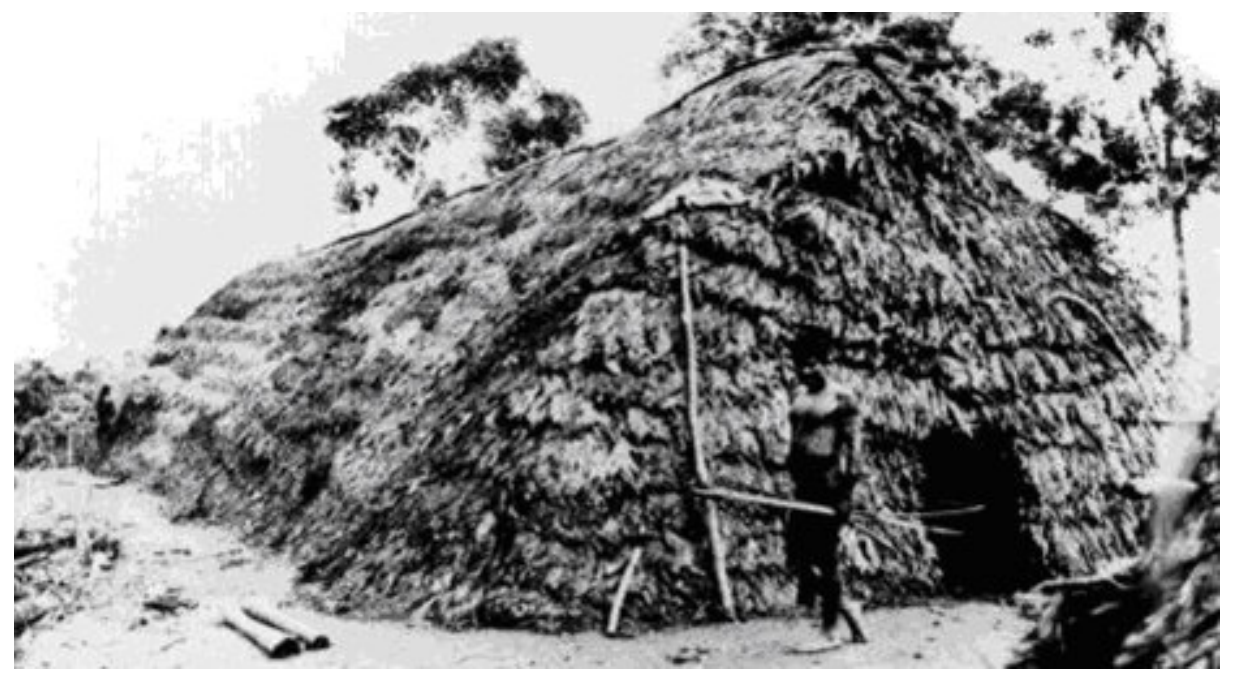

Foto 1: Vivienda Autóctona Amarakaeri (1953).

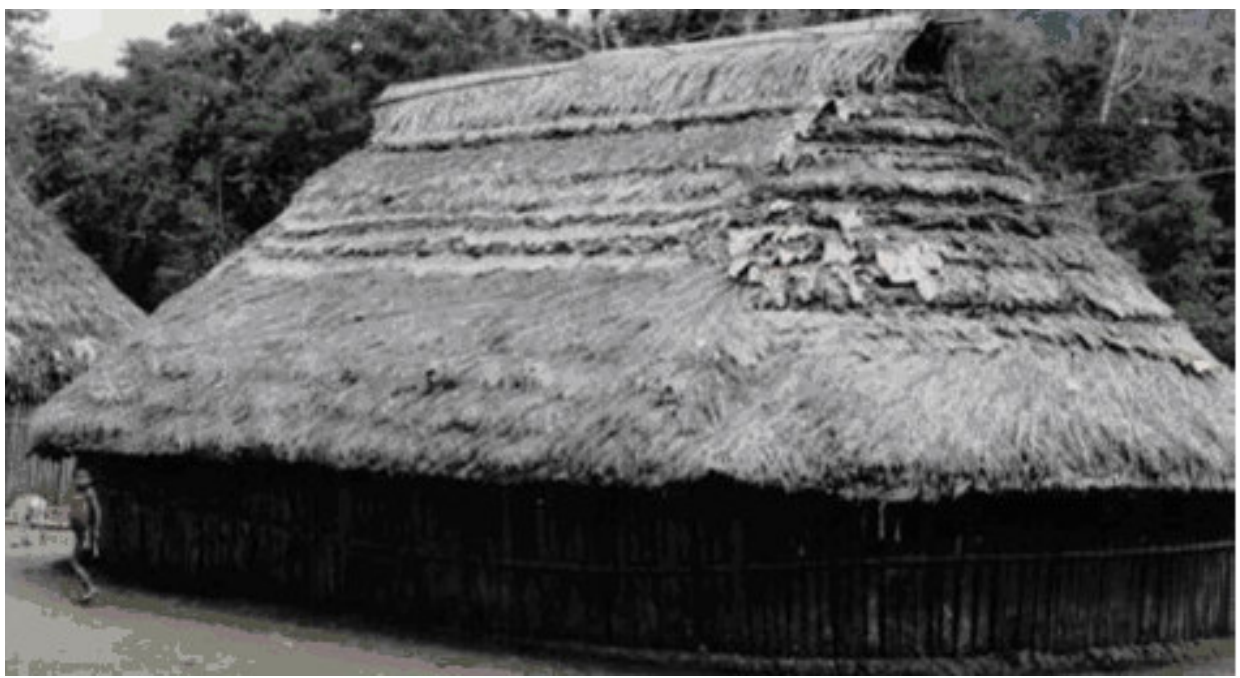

Foto 2: Vivienda Tradicional Matsiguenka.

Fuente: Nishiyama y Olórtegui del Castillo).

- Viviendas Tradicionales (T): Son viviendas construidas con materiales que poseen tradición de uso en la zona. Los materiales tradicionales en la Amazonía son la madera rolliza para las estructuras, las hojas de palmera para cubiertas, madera en "rajas" para los cerramientos o piel y "pona batida" para los pisos elevados, uniones amarradas o clavadas. Son viviendas unifamiliares de planta rectangular y piso elevado; el espacio interior es uno solo de uso múltiple o de dos divisiones. Generalmente la cocina constituye otra edificación separada de la casa-habitación. Estas viviendas dependen 
de un mínimo de materiales foráneos y herramientas como machetes y hachas. Representa el 24\% del total de muestras estudiadas y se encuentran en las comunidades San José del Karene, Boca del Inambari, Infierno, Diamante y Puerto Arturo.

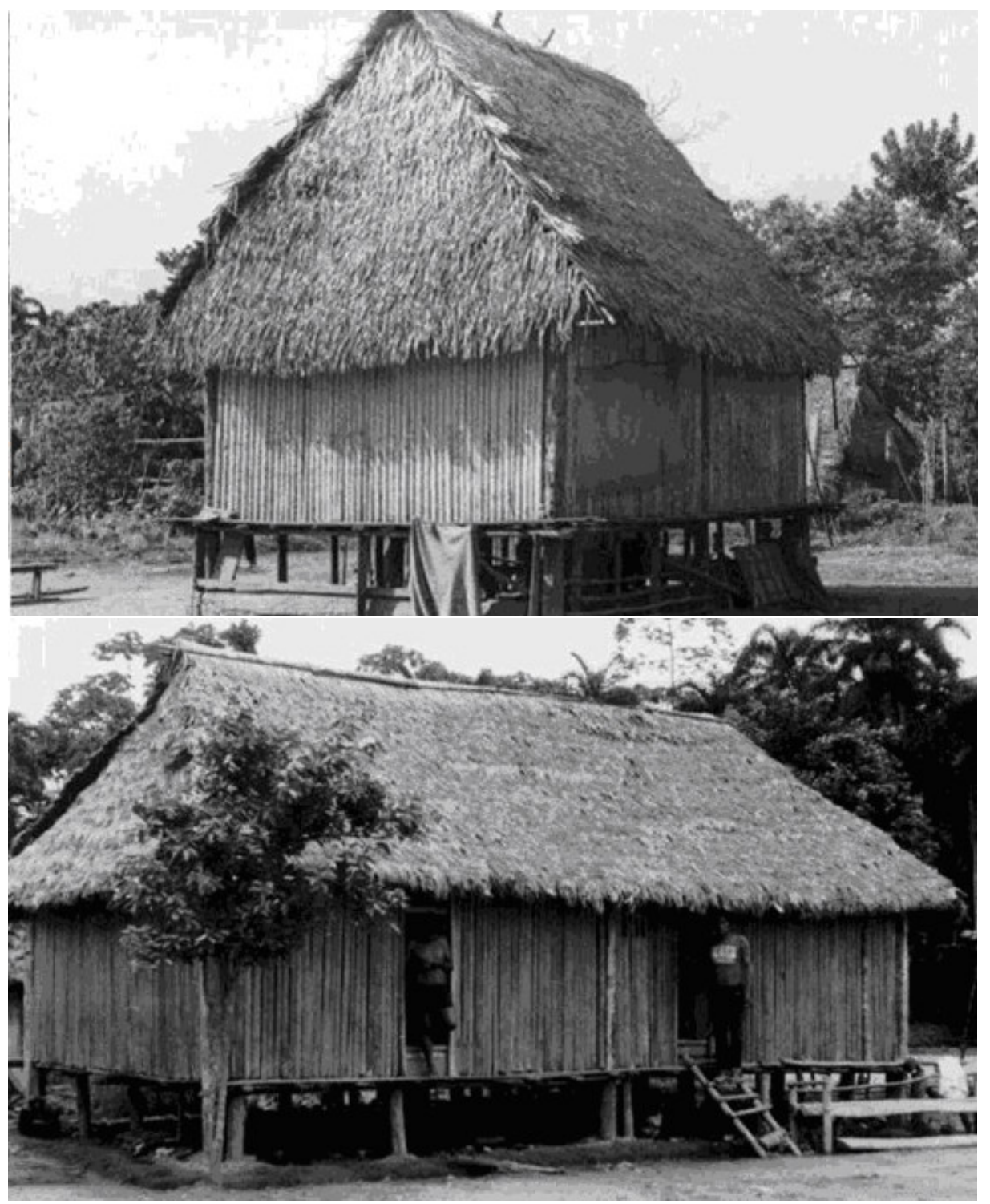

Foto 3 y 4: Viviendas Tradicionales (T) Amarakaeri. Fuente: Olórtegui del Castillo.

- Viviendas en Transición (TAT, TTM): Son viviendas autóctonas o tradicionales modificadas en su forma, piel y/o en su organización 
espacial; presentan nuevos materiales que sustituyen a los originales. Son también viviendas que se construyen desde el comienzo con una mezcla indiscriminada de materiales autóctonos, tradicionales y modernos.

Las Viviendas en Tránsito Autóctona Tradicional (TAT), son las que presentan La máxima autonomía en cuanto a oferta de materiales y no requieren de recursos financieros para su ejecución. Existen sólo en Palotoa-Teparo aunque en su mayoría construidas com algunos elementos procesados (tablas), pero su distribución espacial responde aún a patrones propios de su cultura. Representan el $2 \%$ del total estudiado .

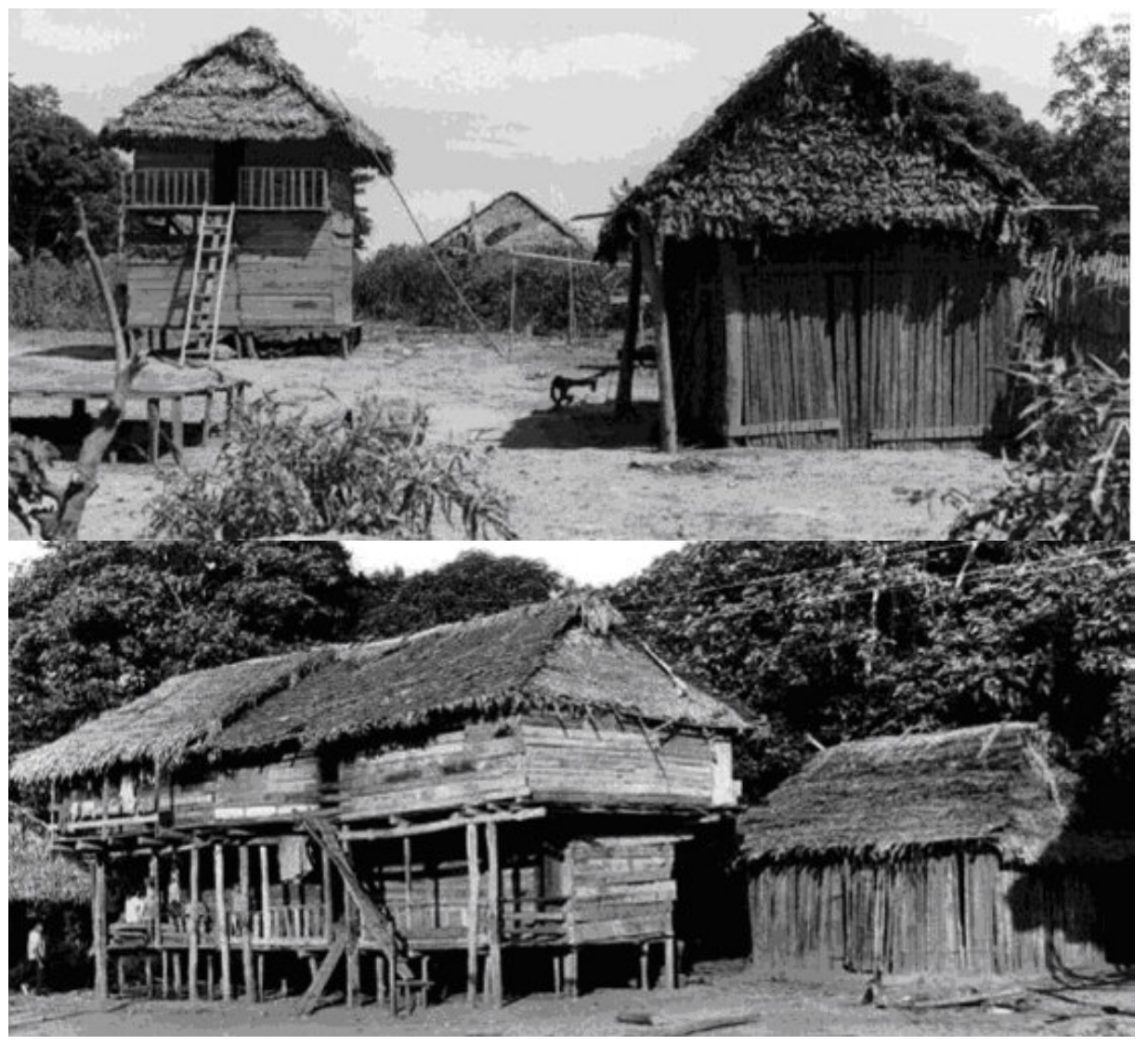

Foto 5 y 6: Viviendas en Tránsito (TAT).

Fuente: Olórtegui del Castillo

\footnotetext{
${ }^{1}$ El total estudiado está compuesto por 46 viviendas. este total significa el $23 \%$ del universo de viviendas existente en las comunidades nativas mencionadas en este estudio, es decir 196 viviendas.
} 
- Viviendas Modernas (M): Son aquellas viviendas que se construyen con materiales de origen industrial (calamina, clavos, etc.) y madera cortada (tablas) con motosierras. También se considera las que presentan cambios en su distribución interior asemejándose a las viviendas urbanas. Por lo general son viviendas unifamiliares de planta rectangular, piso elevado o de tierra apisonada. Estas viviendas son costosas dado que ya no utilizan recursos inmediatos y exigen gastos de transporte para el traslado de materiales a las comunidades y herramientas como motosierras y aserraderos portátiles que requieren combustible para su funcionamiento. Representan el 9\% del total de viviendas estudiadas.

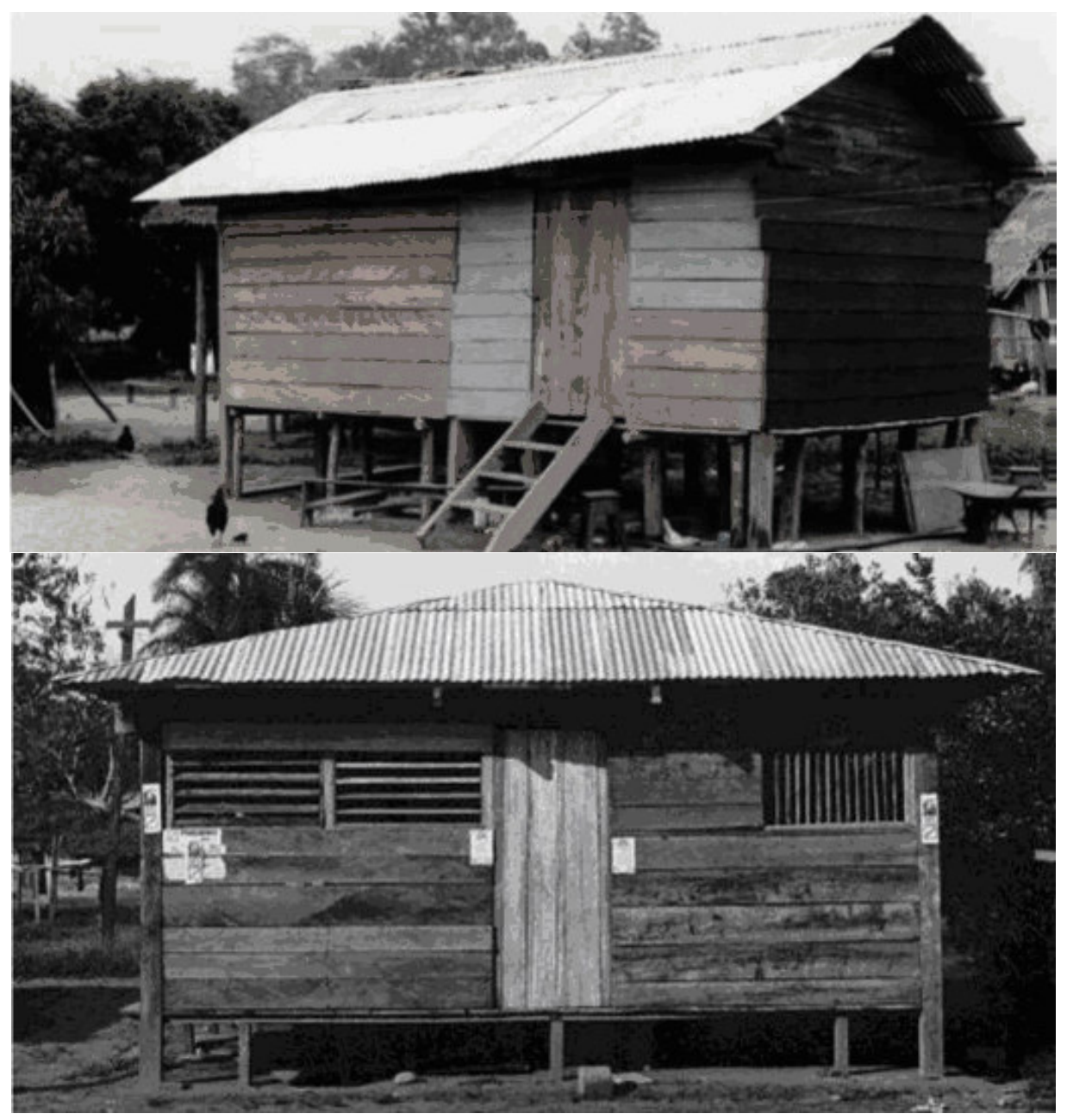

Foto 7 y 8: Viviendas Modernas con techos de 2 y 4 aguas. Fuente: Olórtegui del Castillo. 


\section{ANALIZANDO LA VIVIENDA INDÍGENA}

Nuestro análisis se centra en los aspectos formales y espaciales característicos de la vivienda indígena, resaltando la connotación de los mismos para un mejor entendimiento del cosmos y modo de vida de la población indígena.

Los componentes de la unidad de habitación indígena están localizados dentro del predio de tal forma que cumplen con las actividades que requiere la vida de la familia. La extensión de dicha unidad es mayor que las edificaciones que la componen; existe un espacio habitable de gran importancia que es el que rodea las edificaciones delimitando el alcance de lãs actividades domésticas siendo a la vez un espacio de relación social.

En algunos casos estudiados, el espacio exterior de la vivienda es un espacio poço saneado, principalmente por la introducción de la crianza de cerdos que deambulan alrededor de las viviendas; además la existencia de residuos no orgánicos como plásticos y latas de conservas que son tirados entre los árboles y plantas cercanas.

El control ambiental de las edificaciones es significativo porque demuestra la mayor o menor adecuación interna de la vivienda, siendo la construcción de las edificaciones uno de los factores que más afecta a ésta. Por ejemplo, el material de los cerramientos de las habitaciones define su aptitud para el control climático. Las paredes de rajas de huasaí o pona son aptas para el clima cálido húmedo ya que permiten el paso del aire por los intersticios de separación, al igual que los techos de hojas de palmera, de gran permeabilidad al paso del aire. Los câmbios ocasionados en las edificaciones son generalmente insatisfactorios desde el punto de vista ambiental, ya que al usar materiales procesados pero sin una tecnología apropiada como tablas superpuestas y calaminas sin ningún tipo de aislamiento en los techos, las cuales producen um efecto de cerramiento demasiado hermético evitando una adecuada ventilación e iluminación. Es preciso considerar alternativas tecnológicas que tomen en cuenta estos aspectos.

Las cocinas por estar construidas en su mayoría con materiales del lugar y com tecnología tradicional tienen características ambientales favorables. Son espacios bien ventilados pero con escasa iluminación. Los pisos de tierra apisonada tienen la desventaja de absorber la humedad en los períodos lluviosos; lo cual no sucede con los pisos de madera elevados, sin embargo en esta alternativa es importante considerar el aislamiento del fogón creando una base de tierra o barro donde apoyar los leños y evitar incendios. 
Las edificaciones en muchos casos son deficientes en cuanto a su estabilidad y acabado (por ejemplo viviendas de dos niveles en Palma Real y San José del Karene), hay desconocimiento en lo referente a estructuras más complejas así como de ciertos requisitos indispensables para mejorar la habitabilidad de las viviendas.

Así mismo existen otros problemas ambientales que son más difíciles de remediar sin una asistencia técnica adecuada; pero es preciso recurrir a tecnologías propias de su cultura como en el caso del tratamiento de hojas de palmeras y maderas para evitar el ataque de insectos nocivos. También es necesario mejorar los medios de abastecimiento y purificación de agua, disposición de residuos y ubicación de silos o letrinas de diseño adecuado donde sea necesario. Para ello se requiere un desarrollo tecnológico intermedio accesible al nativo que se integre dentro de su cultura mediante una comprensión de los beneficios que de ella puede obtener.

Las viviendas en transición son el reflejo de la desaparición de ciertas técnicas y materiales tradicionales así como de la confusión cultural en la que las aspiraciones de mejoramiento tienen como meta el cambio de materiales y la "creación" (en algunos casos copia) de espacios en la vivienda, que significan un mayor prestigio para sus dueños. Estas viviendas representan el 53\% del total de casos estudiados, siendo significativa su presencia em las comunidades Palma Real (el 100\% son del estado Tránsito Tradicional Moderno-TTM), Infierno, Diamante, Boca Pariamanu y en menor grado en San José del Karene, Boca del Inambari, Shintuya y Puerto Arturo.

Pero la solución no radica en materiales (ver cuadro 1) y técnicas constructivas tradicionales. Cada comunidad presenta un nivel tecnológico distinto y por tal motivo precisa aportes según sus exigencias; por ejemplo en Palotoa-Teparo las viviendas tienen un estado tecnológico Tránsito Autóctona Tradicional (TAT) y en Shintuya la mayoría posee un estado tecnológico Moderno (M).

Así mismo cabe mencionar que en las comunidades Harákmbut se observa mayores cambios formales y los únicos ejemplos de viviendas modernas, siendo los Ese'Eja quienes demuestran una mayor búsqueda espacial en el interior de sus viviendas y acabados constructivos. También merece especial atención el caso de la comunidad Kichwa Runa que presenta detalles muy finos de estantería adosada a las paredes; óptimamente logrados en cuanto a estructura y forma, siendo a la vez únicos en Madre de Dios. 
Cuadro 1: Especies regionales utilizadas en la construcción de las viviendas.

\begin{tabular}{|c|c|c|c|c|}
\hline \multicolumn{2}{|c|}{$\begin{array}{l}\text { PARTES O ELEMENTOS } \\
\text { DE LA CONSTRUCCIÓN }\end{array}$} & \begin{tabular}{|c|} 
NOMBRE \\
VERNACULAR \\
\end{tabular} & FAMILIA & $\begin{array}{l}\text { GENERO Y } \\
\text { ESPECIE } \\
\end{array}$ \\
\hline \multirow{7}{*}{$\begin{array}{l}\text { In } \\
\text { 党 } \\
0 \\
0 \\
0 \\
0\end{array}$} & \multirow[t]{2}{*}{ TECHO } & PINTANA & ANONACEAE & \begin{tabular}{|l} 
XILOPIA \\
sp./OXANDRA \\
MEDIOCRIS \\
\end{tabular} \\
\hline & & CASHA-PONA & $?$ & SOCRATEA sp. \\
\hline & \multirow{3}{*}{ HORCONES } & HUACAPÚ & OLACACEAE & HEISTERIA sp. \\
\hline & & CUMALA & MYRISTICACEAE & $\begin{array}{l}\text { IRYANTHEA } \\
\text { sp./OTOBA } \\
\text { PARVIFLORA } \\
\end{array}$ \\
\hline & & ESTORAQUE & FABACEAE & $\begin{array}{l}\text { MYROXYLON } \\
\text { BALSAMUN } \\
\end{array}$ \\
\hline & \multirow[b]{2}{*}{ PISO } & HUACAPÚ & OLACACEAE & HEISTERIA sp. \\
\hline & & PINTANA & ANONACEAE & $\begin{array}{l}\text { XILOPIA } \\
\text { sp./OXANDRA } \\
\text { MEDIOCRIS } \\
\end{array}$ \\
\hline \multirow{7}{*}{ 息 } & \multirow{2}{*}{ TECHO } & SHAPAIA & PALMAE & SCHEELA \\
\hline & & PALMICHI & PALMAE & GEONOMA sp. \\
\hline & \multirow[b]{3}{*}{ PAREDES } & HUASAÍ & PALMAE & EUTERPE sp. \\
\hline & & PONA & PALMAE & IRIARTEA sp. \\
\hline & & $\begin{array}{l}\text { CEDRO MACHO } \\
\text { (en el caso de } \\
\text { tablas) } \\
\end{array}$ & ANACARDIACEAE & TAPIRIRIA sp. \\
\hline & CERCOS & CAÑA BRAVA & GRAMINAE & $\begin{array}{l}\text { GYNERIUM } \\
\text { SAGITTATUM } \\
\end{array}$ \\
\hline & PISO & PONA (batida) & PALMAE & IRIARTEA sp. \\
\hline UNIONES & & TAMSHI & ARACEAE & HETEROPSIS sp. \\
\hline
\end{tabular}

NOTA: Cabe mencionar que estas especies son utilizadas tanto en la construcción de viviendas tradicionales ( $\mathrm{T}$ ) como en las de transición tradicional moderno (TTM) y modernas (M). La diferencia radica en el procesamiento del material. en el caso de lãs viviendas tradicionales los elementos de construcción se presentan en su estado natural (como madera rolliza y cortada en rajas). En los otros casos dichos elementos se presentan aserrados (horcones, postes y vigas de sección cuadrada así como tablas).

FUENTE: Autora.

Así mismo cabe mencionar que en las comunidades Harákmbut se observa mayores cambios formales y los únicos ejemplos de viviendas modernas, siendo los Ese'Eja quienes demuestran una mayor búsqueda espacial en el interior de sus viviendas y acabados constructivos. También merece especial atención el caso de la comunidad Kichwa Runa que presenta detalles muy finos de estantería adosada a las paredes; óptimamente logrados en cuanto a estructura y forma, siendo a la vez únicos en Madre de Dios.

Las tipologías organizativas de la vivienda nativa son básicamente dos: T-1: Casacocina (vivienda de un volumen) y T-2: Casa y Cocina 
(vivienda de dos volúmenes); predominando ésta última (ver cuadro $\mathrm{N}^{\mathrm{o}}$ 2). Alrededor de los volúmenes principales existen pequeñas edificaciones utilizadas como gallineros o depósitos, los cuales tienen muchas veces características comunes a la vivienda tradicional, con respecto a forma y materiales.

Dentro de estas tipologías básicas se presentan variantes en cuanto a compartimentación espacial horizontal (de uno o más ambientes) y vertical (uno o dos niveles), siendo esta última variante poco frecuente pero de sumo interés por la alternativa de cambio que representa dentro de los patrones espaciales y formales nativos. Es sin duda un cambio originado por la copia de formas existentes en los poblados mestizos, sin embargo muchos de ellos son desarmados al poco tiempo de construidos porque las familias no logran adaptarse a estas estructuras, debido a que el espacio arquitectónico creado no corresponde a su espacio existencial.

Las composiciones axiales existentes no tienen una norma fija, se observa que tanto lãs composiciones lineales, paralelas y ortogonales presentan un acceso claro desde la trocha de circulación principal. El volumen de la casa-habitación generalmente se ubica próximo a la via de acceso principal quedando las demás edificaciones retiradas o detrás de éste.

En planimetría hay predominio del rectángulo como forma básica, con pocas variantes en "T" y "L". En volumetría son interesantes las variantes que presentan los volúmenes com cubiertas de calamina, a dos, cuatro y una sola agua; siendo la cubierta a dos aguas común em volúmenes construidos con hojas de palmera. Existen formas incompletas que presentan adiciones por algunas caras del volumen.

Un aspecto más particular y difícil de captar ha sido la noción de uso y la disposición de las partes de la vivienda. La manera como delimita su espacio (ya sea virtual o real) demuestra cómo el nativo aprecia su espacio habitable. Lo privado y lo público se presentan cerrado y abierto respectivamente en diversos grados. La delimitación física de áreas reservadas a corrales es consecuencia de la creciente importancia de la crianza de animales domésticos que causan problemas al deambular por el asentamiento.

\section{CONCLUSIONES Y SUGERENCIAS EN EL CAMPO DE LA VIVIENDA INDÍGENA}

No pretendemos aquí normar el diseño y la construcción de las viviendas en comunidades nativas; consideramos que éstas deben surgir 
del contexto cultural de cada grupo étnico, únicamente sugerimos ciertas pautas generales que contribuirían a mejorar las condiciones de habitabilidad de la vivienda indígena, favoreciendo el bienestar de la población indígena, rescatando así mismo aspectos de la relación de la vivienda indígena con el medio ambiente, su tecnología constructiva y su arquitectura, reforzando a partir de la valorización de estos aspectos de su cultura material la identidad de las culturas indígenas amazónicas.

Estas pautas serían las siguientes:

- Es preciso respetar el área libre circundante a la vivienda, donde se desarrollan actividades sociales y se ubican locales anexos como gallineros y depósitos.

- Con respecto al uso de materiales en la construcción, se recomienda preferir la madera como material principal buscando darle un tratamiento adecuado para hacerla más durable; evitar en lo posible el uso de materiales ajenos a la zona tanto por razones económicas como de adaptación climática.

- Por razones de ventilación, la vivienda no debería estar adosada lateralmente a otras edificaciones; de igual manera se debería elevar en lo posible los volúmenes del suelo para evitar la humedad de la tierra.

- Las envolventes, sea cual fuere su material y estado tecnológico, deberían ser permeables al paso del aire.

- Igualmente es aconsejable mantener las proporciones armónicas de las viviendas (que coinciden con la proporción áurea o proporción de oro de los clásicos) así como lãs inclinaciones de los techos (ya que éstos garantizan un mayor confort debido al mayor volumen de aire que albergan en la parte superior, dejando el espacio habitable siempre fresco). Como datos, se recomienda las siguientes proporciones en las plantas a $/ \mathrm{L}=1 / 1.6=1 / 2$; altura promedio de piso a techo 2.50-3.00 m.; altura promedio del suelo al piso elevado 1.00 m.y ángulos de inclinación de techos mayores o iguales a $45^{\circ}$.

Finalmente consideramos estas pautas como un aporte hacia la creación de vías alternativas de desarrollo sostenido desde valores endógenos de las culturas tradicionales y como propuestas viables de ser ejecutadas desde y para los pueblos amazónicos combinando el conocimiento local con los avances de la técnica y la investigación modernos. Apostamos aquí por una visión que enfrente la búsqueda de soluciones partiendo desde nuestros propios valores. 


\section{REFERÊNCIAS}

ARIZ, Javier. Ven a mi rio. In: Misiones Dominicanas del Perú. Lima, v. XXXIX, n. 222, p. 195-200, 1957.

BARRIALES, Joaquín; TORRALBA, Adolfo. Los mashcos hijos del Huanamei. Lima: Secretariado de Misiones Dominicanas del Perú, 1970.

CALIFANO, Mario. Etnografía de los Mashco de la Amazonía Sudoccidental del Perú. Buenos Aires: Fundación para la Educación, la Ciencia y la Cultura, 1982.

CORNEJO BOURONCLE, Jorge. Los indios Machcos (Perú). In: Revista Geográfica Americana, v. IX, n. 177. Buenos Aires, 1943.

CHAUMEIL, Jean Pierre. ÑIHAMWO, Los Yagua del nor-oriente peruano. Lima: Centro Amazônico de Antropología y Aplicación Práctica. 1984.

CHAVARRIA M., Maria C. Términos de parentesco y organización clánica eséeja. Lima: Universidad Mayor de San Marcos UNMSM, Centro de Lingüística Aplicada. Documento de Trabajo n. 49, 1984.

FARABEE, William Curtis. Indian Tribes of Eastern Peru. In: Papers of the Peabody Museum of American Archaeology and ethnology and Ethnology, vo. X. Cambridge, Mass.: Harvard University, 1922.

FERSTL, Edmund. Die Machiguenga im Parque Nacional Manu, Madre de Dios (Südostperu). Tesis de Doctorado, Universidad de Viena. Viena, Austria, 1984.

FONSECA MARTINEZ, Lorenzo; SALDARRIAGA, Alberto. La arquitectura de la vivienda rural en Colombia. Bogotá, Colombia: Colciencias, v. 1, 1980.

GRAIN, José Maria. La vivienda Machiguenga. In: Misiones Dominicanas del Perú, v. XX, n. 106, p. 92-93, Lima.

GRAY, Andrew. Y después de la fiebre del oro...? Derechos humanos y autodesarrollo del sudeste Del Perú. Copenhage: Documento IWGIA n. 5, 1986.

. Los Amarakaeri: una noción de estructura social. In: Amazonía Peruana, v. V, n. 10, p. 47-63, Lima, 1984.

. The Amarakaeri: An Ethnographic Account of Harákmbut people from Southeastern Peru. Tesis de Doctorado, Oxford: Universidad de Oxford, UK, 1983.

HOLZMANN, Günther. La tribu mashca. In: Misiones Dominicanas del Perú, v. XXXV, n. 194, p. 17- 19, Lima, 1953.

NORBERG-SCHULZ, Christiam. Existencia, Espacio y Arquitectura. Londres UK: Seudovista, 1971. 
NORDENSKIÖLD, Erland. Beiträge zur Kenntnis einiger Indianerstämme des Rio Madre de Dios Gebietes. In: Ymer, n. 25, p. 265-312, Estocolmo, 1905.

OFICINA NACIONAL DE EVALUACIÓN DE RECURSOS NATURALES (ONERN). Inventario, evaluación e integración de los recursos naturales de la zona de los ríos Inambari y Madre de Dios. Lima: ONERN, 1972.

OLÓRTEGUI DEL CASTILlO, Tanith. Estudio de los patrones de asentamiento y vivienda Del grupo Yagua (Selva Baja). Tesis de Bachiller en Arquitectura, Universidad Ricardo Palma, Lima, 1985.

Centro Etnobiológico Ametra 2001. Tesis de Título Profesional en Arquitectura. Lima: Universidad Ricardo Palma, Facultad de Arquitectura y Urbanismo, 1987.

OLÓRTEGUI DEL CASTILLO, Tanith; RUMMENHÖLLER, Klaus. Cambios en la arquitectura de lãs viviendas de grupos nativos en Madre de Dios. El caso de la comunidad nativa Boca del Inambari. In: Perú Indígena, v. 12, n. 28. Lima: Instituto Indigenista Peruano, 1990.

RIOS, Manuel A., et al. Plan maestro, Parque Nacional del Manu. Lima: Universidad Nacional Agraria La Molina, 1986.

ROSSI, Aldo. Arquitectura. Madrid: Edición Alianza, 1979.

RUMMENHÖLLER, Klaus; CÁRDENAS, Clara; LAZARTE, Maria. Diagnóstico Situacional de Comunidades Nativas de Madre de Dios. Propuestas para un autodesarrollo. Lima: Instituto Indigenista Peruano, 1991.

SZYSLO, Vicente. La arquitectura de los piros. In: Extracta n. 3, p. 6-12. Lima, 1985.

. Los asentamientos piro y la persistencia de su identidad. In: Anthropológica, n. 3, p. 143-152. Lima: Pontificia Universidad Católica del Perú, 1985.

VELLARD, Jehan. Los indios guarayos del Madre de Dios y del Beni. In: Boletín del Instituto Riva Agüero, n. 10, p. 137-167. Lima, 1977.

WAHL, Lissie; RUMMENHÖLLER, Klaus. La Región del Madre de Dios, Bibliografía Anotada. Cusco Perú: Centro de Estudios Regionales Andinos "Bartolomé de las Casas", 1991.

ZELENY, Minislav. Contribución a la etnografía y clasificación del grupo étnico huarayo (ece’je), Madre de Dios, Perú. Praga: Universita Korlova Praha, 1976. 